\title{
Polar Coordinate Drawing of Planar Graphs with Good Angular Resolution
}

\author{
Christian A. Duncan ${ }^{1}$ and Stephen G. Kobourov ${ }^{2}$ \\ 1 Department of Computer Science \\ University of Miami \\ Coral Gables, FL 33124 \\ duncan@cs.miami.edu \\ 2 Department of Computer Science \\ University of Arizona \\ Tucson, AZ 85721 \\ kobourov@cs.arizona.edu
}

\begin{abstract}
We present a novel way to draw planar graphs with good angular resolution. We introduce the polar coordinate representation and describe a family of algorithms which use polar representation. The main advantage of using a polar representation is that it allows us to exert independent control over grid size and bend positions. Polar coordinates allow us to specify different vertex resolution, bend-point resolution and edge separation. We first describe a standard (Cartesian) representation algorithm (CRA) which we then modify to obtain a polar representation algorithm (PRA). In both algorithms we are concerned with the following drawing criteria: angular resolution, bends per edge, vertex resolution, bend-point resolution, edge separation, and drawing area.

The CRA algorithm achieves 1 bend per edge, unit vertex and bend resolution, $\sqrt{2} / 2$ edge separation, $5 n \times \frac{5 n}{2}$ drawing area and $\frac{1}{2 d(v)}$ angular resolution, where $d(v)$ is the degree of vertex $v$. The PRA algorithm has an improved angular resolution of $\frac{\pi}{4 d(v)}, 1$ bend per edge, and unit vertex resolution. For the PRA algorithm, the bend-point resolution and edge separation are parameters that can be modified to achieve different types of drawings and drawing areas. In particular, for the same parameters as the CRA algorithm (unit bend-point resolution and $\sqrt{2} / 2$ edge separation), the PRA algorithm creates a drawing of size $9 n \times \frac{9 n}{2}$.
\end{abstract}

\section{Introduction}

In the area of planar graph drawing there has been considerable interest in algorithms that produce readable drawings 4. Among the many properties which contribute to the readability of planar graphs, edge smoothness, vertex resolution, bend-point resolution, angular resolution, and edge separation are of great importance. Edges are often drawn as straight-line segments connecting two vertices. An edge can also be drawn as a sequence of straight-line segments, in which case the smallest number of bends is desirable. An edge may also be drawn as a smooth curve. These three types of edges generally provide aesthetically pleasing drawings.

P. Mutzel, M. Jünger, and S. Leipert (Eds.): GD 2001, LNCS 2265, pp. 407-421, 2002.

(C) Springer-Verlag Berlin Heidelberg 2002 


\subsection{Definitions}

A graph drawing has good vertex resolution if vertices cannot get arbitrarily close to one another, that is, if vertices are well distributed in the drawing. As a result, a great deal of research has been concentrated on graph drawing algorithms which place vertices on the integer grid such that the drawing area is proportional to the number of vertices $n$ of the graph, typically $O(n) \times O(n)$. If there are bends in the edges, then the bend-points are also placed on the integer grid. The bendpoint resolution of a graph refers to the minimum distance between two bends. The edge separation of a graph refers to the minimum distance between two edges that are sufficiently away from their endpoints (since incident edges can get arbitrarily close to each other near their common endpoint).

A graph drawing has good angular resolution if adjacent edges cannot form arbitrarily small angles. This is achieved by ensuring that the edges emanating from a given vertex "fan out" evenly around the vertex. Note, however, that good angular resolution cannot always be achieved while simultaneously guaranteeing straight-line edges and small sub-exponential drawing area 9]. By introducing bends in the edges, however, we can guarantee both good resolution and small drawing area.

\subsection{Previous Work}

Garg and Tamassia [5] consider the problem of drawing with good angular resolution, and Kant [8] shows how to create drawings with angular resolution of $\Theta(1 / d(v))$ in an $O(n) \times O(n)$ area grid, using edges with at most three bends each. Gutwenger and Mutzel [7] describe an improved algorithm with better constant factors which produces very aesthetically pleasing drawings in a $(2 n-5) \times(3 n / 2-7 / 2)$ grid with at least $2 / d(v)$ angular resolution using at most three bends per edge. The algorithm of Goodrich and Wagner [6] requires one less bend per edge and guarantees angular resolution of $\Theta(1 / d(v))$ for each vertex $v$, but at the expense of larger area, $(20 n-48) \times(10 n-24)$. Cheng, Duncan, Goodrich, and Kobourov [1] improve the above algorithm so that every edge has at most one bend while the angular resolution is $\Theta(1 / d(v))$ for each vertex $v$ and maximum area is $30 n \times 15 n$.

\subsection{Our Results}

We first present a new Cartesian representation algorithm (CRA) which improves the bounds of previous algorithms. In particular, CRA guarantees 1 bend per edge, unit vertex resolution, unit bend-point resolution, $\sqrt{2} / 2$ edge separation, $5 n \times \frac{5 n}{2}$ drawing area, and $\frac{1}{2 d(v)}$ angular resolution, where $d(v)$ is the degree of vertex $v$.

We then present a novel polar representation algorithm (PRA). The PRA algorithm also guarantees $\frac{\pi}{4 d(v)}$ angular resolution, 1 bend per edge, and unit vertex resolution. The bend-point resolution and edge separation are parameters that can be modified to achieve different types of drawings and drawing areas. 
In particular, for the same parameters as the CRA algorithm (unit bend-point resolution and $\sqrt{2} / 2$ edge separation), the PRA algorithm creates a drawing of size $9 n \times \frac{9 n}{2}$. Note that in some situations the vertex resolution is more important than the bend-point resolution or the edge separation. In such situations, all of the previous algorithms perform poorly since they are designed to maintain constant resolution particularly between vertices and bend-points. Using the PRA algorithm, we can relax the bend-point resolution constraints and get significant improvements.

The PRA algorithm relies on a novel approach for representing bends and vertices. Traditionally, vertices and bend-points are restricted to lie on integer grid coordinates. One reason for this is that the points are defined by a pair of integers. In this way, all operations on the points (for example, shifting) are performed with integer arithmetic. At the drawing stage, the integer coordinates are mapped to pixels on the screen.

Another reason for placing vertices and bend-points on integer grid coordinates is that this approach guarantees good vertex resolution, good bend-point resolution, and good edge separation [1/6/7/8]. Rather than insisting that bendpoints lie on integer grid coordinates, we propose an alternative approach which allows bend-points to be located on a grid represented by polar coordinates. We call this a polar representation approach because both the vertices and the bend-points are represented using polar coordinates.

At the exact moment of drawing the graph onto the screen, an algorithm using polar representation requires a rounding calculation to determine the exact pixel location for the bend-points. Note, however, that the traditional approach also uses a rounding calculation for scaling from the integer grid space to the pixel space.

The main advantage of using a polar representation is that it allows us to independently control grid size and bend positions. Polar coordinates allow us to specify different vertex resolution, bend-point resolution, and edge separation. We achieve this added flexibility at the expense of slightly increased storage for the graph representation. A Cartesian representation requires exactly two integers for each point while the polar representation requires up to five integers per point.

\section{The CRA Algorithm}

The Cartesian Representation Algorithm is a natural extension of the previous algorithms which guarantee good angular resolution [8]76,1]. For the remainder of this paper, when we say "graph" we mean a fully triangulated, undirected, planar graph. In our algorithm the vertices of the graph are inserted sequentially by their canonical ordering, generating subgraphs $G_{1}, G_{2}, \ldots, G_{n}$. The canonical ordering [3] for a planar graph $G$ orders the vertices of $G$ so that they can be inserted one at a time without creating any crossings. We define $G_{k}$ at step $i$ to be the graph induced by vertices $1,2, \ldots, k$. Graph $G_{k+1}$ is created from $G_{k}$ by inserting the next vertex $v_{k+1}$ in the canonical order. Before we show the details 


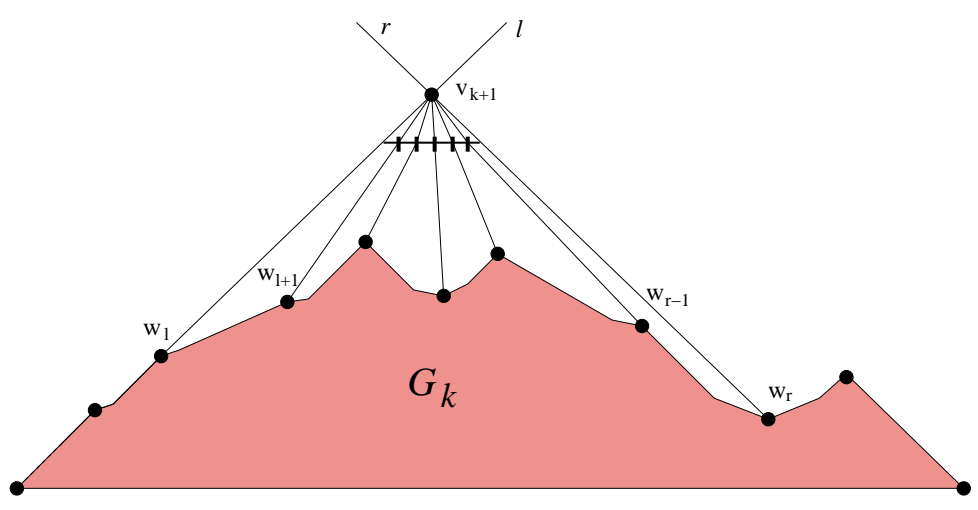

Fig. 1. Graph $G_{k+1}$ after inserting $v_{k+1}$. The shaded part is $G_{k}$. Vertices $w_{l}$ and $w_{r}$ are the leftmost and rightmost neighbors of $v_{k+1}$. The horizontal line segment below $v_{k+1}$ is the middle port region through which all the edges $\left(v_{k+1}, w_{i}\right), l<i<r$, are routed.

of our algorithm we need several definitions. Following the notation of [3], let $w_{1}=v_{1}, w_{2}, \ldots, w_{m}=v_{2}$ be the vertices of the exterior face $C_{k}$ of graph $G_{k}$ in order. For a particular subgraph $G_{k}$ and vertex $v_{k+1}$, we refer to $w_{l}$ and $w_{r}$ as the leftmost and rightmost neighbors of $v_{k+1}$ on $C_{k}$, see Fig. 1]

\subsection{Vertex Regions}

In the immediate vicinity of every vertex there are two types of regions: free regions and port regions. The free and port regions alternate around the vertex, see Fig. 2(a). For each free region there is at most one edge passing through it to $v$. Each port region is bounded by a line segment with a number of ports and every edge inside the port region passes through a unique port. The number of ports in a port region is as small as possible. Define and name the six regions around $v$ as follows:

There are three free regions $M^{f}$ (between $-45^{\circ}$ and $45^{\circ}$ ), $R^{f}$ (between $90^{\circ}$ and $135^{\circ}$ ), and $L^{f}$ (between $-135^{\circ}$ and $-90^{\circ}$ ). There are also three port regions $M^{p}$ (between $L^{f}$ and $R^{f}$ ), $L^{p}$ (between $L^{f}$ and $M^{f}$ ), and $R^{p}$ (between $R^{f}$ and $\left.M^{f}\right)$.

The algorithm draws each edge in $E$ by "routing" it through a port of one of the two vertices in a fashion similar to [1. Each edge consists of two connected edge segments. One edge segment, the port edge segment, connects a vertex with one of its ports. The other segment, the free edge segment, connects a vertex to one of its neighbor's ports. For example, for an edge $e=(u, v)$, if we route $e$ through the leftmost port in $u$ 's middle port region $M^{p}$, we would draw two line segments, see Fig. 2(b): the port edge segment would pass from $u$ to the port, and the free edge segment would pass from the port to $v$. This method of 


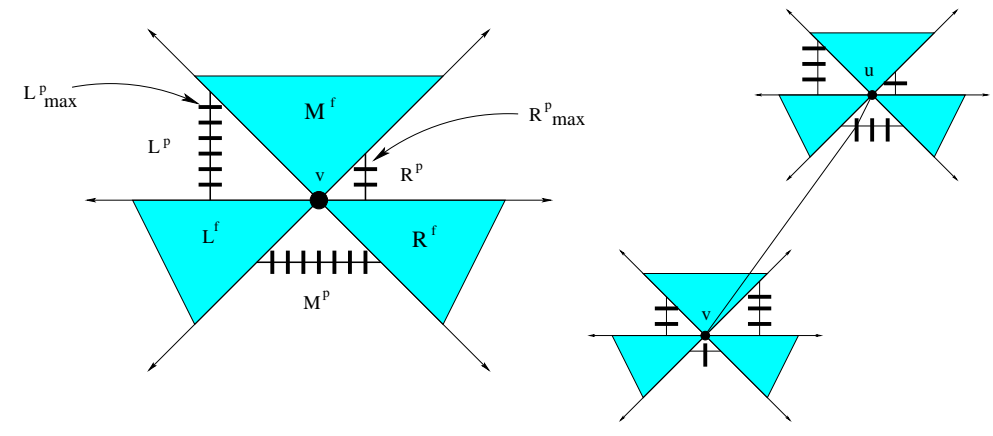

Fig. 2. Vertex regions and edge routing: the number of ports along each port region is determined by the number of edges that need to be routed through that port region. (a) The area around a vertex $v$ is divided into 6 regions. The free regions are shaded and at most one free edge segment goes through each one of them. All the port segments use ports in the port regions of $v$. (b) Routing an edge $e=(u, v)$, where the port edge segment connects $u$ to one of its ports and the free edge segment connects the port to $v$, going through one of $v$ 's free regions.

construction guarantees that the free edge segments always pass through free regions and that each port transmits at most one port edge segment.

We perform our construction in incremental stages, where each stage corresponds to the insertion of a new vertex. Observe that at each stage, for every vertex $v$ except those on the external face, $w_{1}=v_{1}, w_{2}, \ldots, w_{m}=v_{2}$, there are exactly three free edge segments. The remaining edges are connected to $v$ via port segments. These remaining edges can be grouped into three classes based on which port region they are routed through, $L^{p}, R^{p}$, or $M^{p}$. Count the number of edges in each of these groups and let $d_{l}(v)$ be the number of port edge segments using port region $L^{p}$. Similarly, define $d_{r}(v)$ and $d_{m}(v)$ to be the number of port segments using port regions $R^{p}$ and $M^{p}$. Observe that in the final stage, there are exactly three vertices on the exterior face, $v_{1}, v_{2}, v_{n}$, and then $\sum_{v \in V}\left(d_{l}(v)+d_{r}(v)+d_{m}(v)\right)=|E|$. That is, for every edge, there is a corresponding port and free edge segment.

For a vertex $v$ we define the maximal right port $R_{\max }^{p}$ as follows. Let $v$ have coordinates $\left(v_{x}, v_{y}\right)$. Then the $R_{\max }^{p}$ of $v$ has coordinates $\left(v_{x}+d_{r}(v)+1, v_{y}+\right.$ $\left.d_{r}(v)\right)$ if $d_{r}(v)>0$ and $\left(v_{x}, v_{y}\right)$ otherwise. We define the maximal left port $L_{\max }^{p}$ of $v$ in a similar fashion, see Fig. 2(a).

\subsection{Invariants of the CRA Algorithm}

By design, our algorithm is incremental with $n$ stages, where each stage corresponds to the insertion of the next vertex in the canonical order. Thus it is natural to define several key invariants to be maintained at every stage. The 
four invariants below are similar in flavor to those of Cheng et al [1] except that here we do not need to maintain any joint boxes.

1. All vertices and ports have integer coordinates.

2. Let $w_{1}=v_{1}, w_{2}, \ldots, w_{m}=v_{2}$ be the vertices of the exterior face $C_{k}$ of $G_{k}$ in order, and let $x\left(w_{i}\right)$ be the $x$-coordinate of vertex $w_{i}$. Then $x\left(w_{1}\right)<x\left(w_{2}\right)<$ $\ldots<x\left(w_{m}\right)$.

3. The free edge segment of edge $e=\left(w_{i}, w_{i+1}\right), 0<i<m$, has slope \pm 1 and $e$ 's port edge segment goes through a maximal port.

4. For every vertex $v$ there is at most one (free) edge segment crossing each of its free regions. All other edge segments are port edge segments.

\subsection{Vertex Shifting}

In the algorithms that maintain good angular resolution with the aid of vertex joint boxes [16], every time a new vertex is inserted, already placed vertices need to be shifted a great deal so that the joint box can fit amongst them. The amount of shifting required is typically of the order of the degree of the vertex. Invariably this leads to large constants behind the $O(n) \times O(n)$ area, e.g. $(20 n-48) \times(10 n-24)$ in [6] and $30 n \times 15 n$ in [1]. In our algorithm we never need to shift any vertex by more than five grid units allowing us to draw $G$ in a $5 n \times \frac{5 n}{2}$ grid. When a new vertex $v$ is inserted, we must create enough space so that the leftmost $w_{l}$ and rightmost $w_{r}$ neighbors of $v$ can "see" $v$ through their respective maximal port regions. Note that the previous $R_{\max }^{p}$ port of $w_{l}$ and $L_{m a x}^{p}$ of $w_{r}$ were used at an earlier stage. Thus, we must create an additional port along the $R^{p}$ region of $w_{l}$. Similarly, additional space is necessary along the $L^{p}$ region of $w_{r}$.

In order to create more space we need to move $w_{l}$ and $w_{r}$. We also have to ensure that the four invariants and the planarity of the graph are maintained. This is achieved by shifting the "shifting set" of the vertex as well as the vertex itself. Using the definition of de Fraysseix et al [3, define the shifting set $M_{k}\left(w_{i}\right)$ for a vertex $w_{i}$ on the external face of $G_{k}$ to be a subset of the vertices of $G$ such that:

1. $w_{j} \in M_{k}\left(w_{i}\right)$ iff $j \geq i$

2. $M_{k}\left(w_{1}\right) \supset M_{k}\left(w_{2}\right) \supset \ldots \supset M_{k}\left(w_{m}\right)$

3. Let $\delta_{1}, \delta_{2}, \ldots, \delta_{m}>0$; if we sequentially translate all vertices in $M_{k}\left(w_{i}\right)$ by distance $\delta_{i}$ to the right $(i=1,2, \ldots, m)$, then the embedding of $G_{k}$ remains planar.

These shifting sets can be defined recursively. Let $w_{l}$ and $w_{r}$ be the leftmost and rightmost neighbors of $v$ on $C_{k}$. Then construct $M_{k+1}\left(w_{i}\right)$ recursively as follows:

$$
\begin{gathered}
M_{k+1}\left(w_{i}\right)=M_{k}\left(w_{i}\right) \cup v_{k+1}, \text { for } i \leq l, \\
M_{k+1}\left(v_{k+1}\right)=M_{k}\left(w_{l+1}\right) \cup v_{k+1}, \\
M_{k+1}\left(w_{j}\right)=M_{k}\left(w_{j}\right), \text { for } j \geq r .
\end{gathered}
$$


For convenience, define a right-shift of $m$ units for a vertex $w_{i}$ as shifting $M_{k}\left(w_{i}\right)$ by $m$ units to the right so that all ports for every vertex in $M_{k}\left(w_{i}\right)$ also shift except the ports in the $L^{p}$ region of $w_{i}$. Define a left-shift of $m$ units for vertex $w_{i}$ as shifting $M_{k}\left(w_{i+1}\right)$ by $m$ units to the right so that all ports for every vertex in $M_{k}\left(w_{i+1}\right)$ also shift. Note that in a left-shift, we also shift the ports in the $R^{p}$ region of $w_{i}$ by $m$ units to the right.

\subsection{CRA Overview}

The CRA algorithm constructs the graph one vertex at a time, by creating the graphs $G_{1}, G_{2}, \ldots, G_{n}$. Constructing $G_{i}, 1 \leq i \leq 3$ is straightforward, so assume that $G_{k}$ has been constructed with exterior face $C_{k}=\left(v_{1}=w_{1}, w_{2}, \ldots, w_{m}=\right.$ $\left.v_{2}\right)$. Suppose we have embedded $G_{k}$ with exterior face $C_{k}$. To construct $G_{k+1}$, let $v_{k+1}$ be the next vertex in the canonical ordering and recall that $w_{l}$ and $w_{r}$ are, respectively, the leftmost and rightmost neighbors of $v_{k+1}$ on the exterior face $C_{k}$.

Recall that $d_{r}\left(w_{l}\right)$ is the current number of port edge segments using $R^{p}$ of $w_{l}$, and that $d_{l}\left(w_{r}\right)$ is the current number of port edge segments using $L^{p}$ of $w_{r}$. There are two cases to consider:

- case (a) $d_{r}\left(w_{l}\right)=0$, see Fig. 3(a).

- case (b) $d_{r}\left(w_{l}\right)>0$, see Fig. 3(b).

In case (a) perform a left-shift of 2 units on $w_{l}$ in order to free space for a port in the $R^{p}$ region of $w_{l}$. In case (b) perform a left-shift of 1 unit on $w_{l}$. Similarly, if $d_{l}\left(w_{r}\right)=0$ then perform a right-shift of 2 units on $w_{r}$. Otherwise perform a right-shift of 1 unit on $w_{r}$.

Insert $v_{k+1}$ at the intersection of lines $l$ and $r$, where $l$ is the line with slope +1 through $w_{l}$ 's maximal right port and $r$ is the line with slope -1 through $w_{r}$ 's maximal left port, see Fig. 1 In the case where lines $l$ and $r$ do not intersect in a grid point it suffices to shift all the elements in $M_{k}\left(w_{r}\right)$ one additional unit to the right.

The edges from $v_{k+1}$ to $w_{l}$ and $w_{r}$ are routed through $w_{l}$ 's maximal right port and $w_{r}$ 's maximal left port, respectively. The remaining edges go from $v_{k+1}$ to vertices $w_{i}, l<i<r$. Let $\left(v_{k+1}(x), v_{k+1}(y)\right)$ be the coordinates of vertex $v_{k+1}$. Let $w_{a}$ be the rightmost vertex such that $w_{a}(x)<v_{k+1}(x)$. Before placing the $M^{p}$ region of $v_{k+1}$ it is necessary to ensure that there are enough ports on it that can be used to connect $v_{k+1}$ to $w_{l}, w_{l+1}, \ldots, w_{r}$. The $M^{p}$ region is a horizontal line segment with $1,3, \ldots, 2 m+1$ ports when the line segment is $1,2, \ldots, m$ grid units below $v_{k+1}$. It is necessary to find how many $w_{i}$ 's are to the left and right of $v_{k+1}$ in order to find exactly how far below vertex $v_{k+1}$, the region $M^{p}$ must be placed. These numbers are $a-l$ and $r-a-1$ respectively (from the definition of $w_{a}$ above). Then place the $M^{p}$ region of $v_{k+1}$ so that its $y$ coordinate is equal to $v_{k+1}(y)-\max (a-l, r-a-1)$. As shown in the next section the $M^{p}$ region can be placed correctly, that is, placed so that it does not intersect the old graph $G_{k}$. After determining the location of $M^{p}$ the edges are 


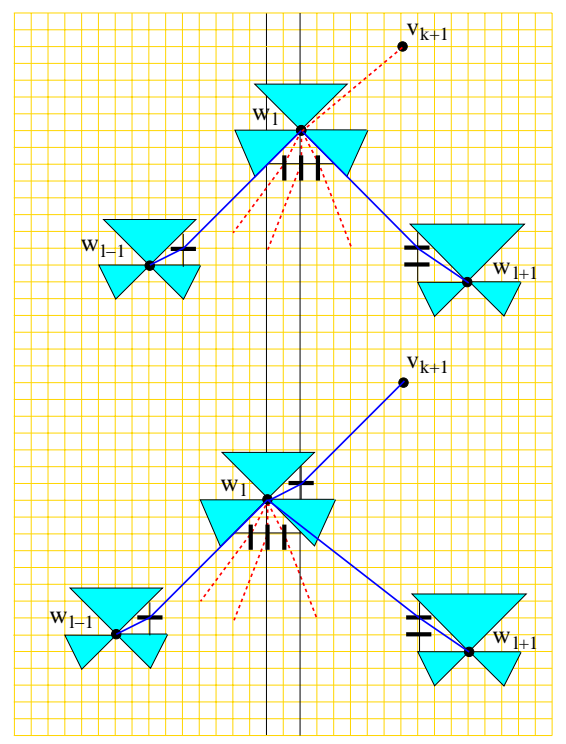

(a)

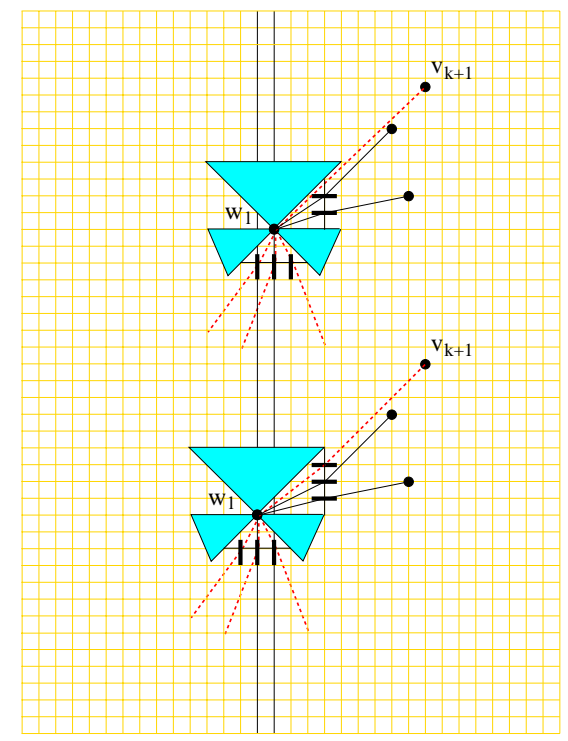

(b)

Fig. 3. Adding the current vertex $v_{k+1}$. Here $w_{l}$ is the leftmost neighbor of $v_{k+1}$ on the exterior face of $G_{k}$. (a) If $d_{r}\left(w_{l}\right)=0$, then we need to shift $w_{l}$ two grid units to the left. (b) If $d_{r}\left(w_{l}\right)>0$, then it suffices to shift $w_{l}$ only one unit to the left. Note that the shifting set $M_{k}\left(w_{l}\right)$ also shifts with $w_{l}$.

routed from $v_{k+1}$ to $w_{l+1}, w_{l+2}, \ldots, w_{a}$ starting from the leftmost port of $M^{p}$ of $v_{k+1}$. Similarly, route the edges from $v_{k+1}$ to $w_{r-1}, w_{r-2}, \ldots, w_{a+1}$ starting from the rightmost port of $M^{p}$ of $v_{k+1}$.

\section{Correctness of the Algorithm}

The algorithm works correctly if all four invariants are maintained. We show that free edge segments always remain in free edge regions and that there is at most one free edge segment per free region. We then need to bound the drawing area required by the algorithm and show that good angular resolution is maintained. Finally, we have to bound the number of bends created and analyze the running time. We leave the detailed proofs for the full version of the paper and present brief proof sketches instead.

Lemma 1. Free edge segments in free regions remain in the free regions.

Proof Sketch: Recall that there are three types of free regions: $M^{f}, L^{f}, R^{f}$. Let us first look at free edge segments in the $M^{f}$ regions. Edge segments in the $M^{f}$ regions are created by a vertex $v$ dominating another vertex $w$. But whenever $v$ dominates $w, w$ is added to the shifting set for $v$ and is only shifted when $v$ is shifted. Therefore, the slope of the free edge segment of the edge connecting $v$ 
and $w$ remains constant and the free edge segment remains within $M^{f}$. As the two remaining cases are symmetric, without loss of generality, let us examine the case when the free edge segment lies in the $L^{f}$ region. This implies that the slope of the free edge segment is between 0 and +1 . Since shifting only moves vertices farther apart in the $x$-direction, the slope can only get closer to 0 , thus remaining in $L^{f}$.

Lemma 2. Every free edge segment passes through a free region which contains no other edges.

Proof Sketch: When a new vertex $v=v_{k+1}$ is inserted there are two types of new edges added: the outside edges between $v$ and the outside neighbors, $w_{l}$ and $w_{r}$, and the inside edges between $v$ and $w_{i}$ where $l<i<r$. In both cases the new edge is routed through a port creating one free edge segment and one port edge segment. A free edge segment of an outside edge has slope either +1 or -1 by construction; therefore it lies inside the free regions $L^{f}$ and $R^{f}$ of vertex $v$. Since $v$ is a new vertex, there are no other segments inside these two free regions.

Dealing with the inside edges is more complex. We first need to show that there is sufficient space between the vertices on the exterior face of $G_{k}$ and the new vertex $v$. Second, we need to show that $v$ has enough ports in its middle port region $M^{p}$ for each of the vertices on $G_{k}$ that it is connected to. Third, we need to show that the free edge segments of the inside edges remain inside their free regions. We begin by showing that for every vertex $w_{i}, l<i<r$,

- vertex $w_{i}$ lies below $M^{p}$, the middle port region for $v$, and

- we can assign a unique port along the $M^{p}$ port region of $v$, such that the edge segment connecting $w_{i}$ to that port fits inside $w_{i}$ 's middle free region $M^{f}$

First consider the vertices $w_{l}, w_{l+1}, \ldots, w_{a}$. In the worst case, they have monotonically increasing $y$-coordinates. Intuitively, this is the worst case because the area of $G_{k+1}-G_{k}$ is the smallest and hence it is more difficult to ensure that there is enough space for the $M^{p}$ region of $v$. Using invariant (3) we can show that $w_{a}(y) \leq v(y)-(a-l)$, since all the edges connecting consecutive vertices on the outer face have both port and free edge segments, thus "freeing" at least one grid point distance between $v$ and $w_{a}$ for every $w_{i}$. Similarly, $w_{a+1}(y) \leq v(y)-(r-(a+1))$.

The same argument implies that there are at least as many ports along the middle port region $M^{p}$ of $v$ as there are vertices $w_{i}, l<i<r$. We route the inside edges for $v$ as follows: starting with the leftmost port on the middle port region $M^{p}$ of $v$, we assign consecutive ports to $w_{l+1}, w_{l+2}, \ldots, w_{a}$ and route the corresponding free edge segments from $w_{i}$ to the ports. The port edge segments for the inside edges connect the same ports to $v$. Similarly, starting with the rightmost port on the middle port region $M^{p}$ of $v$ we assign consecutive ports to $w_{r-1}, w_{r-2}, \ldots, w_{a+1}$ and route the corresponding free edge segments from $w_{i}$ to the ports. The port edge segments for the the inside edges connect the same ports to $v$. 
Combining the above observations, it can also be shown that for every vertex $w_{i}, l<i<r$, the free edge segment of its edge to $v$ fits inside the middle free region $M^{f}$ of $w_{i}$.

Lemma 3. If $G_{k}$ maintains invariants one through four, then $G_{k+1}$ maintains invariants one through four.

Proof Sketch: By definition of the shifting set, invariants one and two hold, see [6]. By construction of the algorithm, invariant three holds as well. Also by construction every edge inserted has a port edge segment and a free edge segment. By lemmas 1 and 2 invariant four also holds.

Lemma 4. The angular resolution for vertex $v \in G$ as produced by the algorithm is $1 / 2 d(v)$, where $d(v)$ is the degree of vertex $v$.

Proof Sketch: The worst angle is achieved between a free edge segment for some edge $f$ and a port edge segment for some edge $e$, where $f$ is located at the boundary of its free region and $e$ is the neighboring port edge segment. There are six possible cases but the argument is the same for all of them, so without loss of generality consider the case in Fig. 4 . Let $v$ be the vertex and $d(v)=d$ its degree. Also let $s$ and $t$ be the lengths as shown in Fig. 4. Let $\theta$ be the angle between $f$ and $e$, and $x$ the number of ports as shown in the figure. Note that all vertices have three edges connected to them via free edge segments. Then the number of ports in any port region is at most $d-3$. From the figure, $\tan (\theta)=t /(s-t)$ and hence $\arctan (t /(s-t))=\theta$. But

$$
\frac{t}{s-t}=\frac{\sqrt{2} / 2}{\sqrt{2}(x+1)-\sqrt{2} / 2}=\frac{1}{2 x+1}
$$

Using the Maclaurin expansion for $\arctan (y)$, where $y<1$ we have

$$
\arctan (y)=y-y^{3} / 3+y^{5} / 5-\ldots
$$

Since $1 /(2 x+1)<1$, and $x \leq d-3$ this yields $\theta \geq 1 / 2 d$ which completes the proof.

Theorem 1. For a given planar graph $G$, the algorithm produces in $O(n)$ time a planar embedding with grid size $5 n \times 5 n / 2$, using at most one bend. The angular resolution for every vertex $v$ of $G$ is $1 / 2 d(v)$.

Proof Sketch: Since every edge has only two segments, there can be at most one bend per edge. Chrobak and Payne [2] show how to implement the algorithm of De Fraysseix, et al. 3 in linear time. Their approach can be easily extended to our algorithm. By invariants three and four and by lemma 4 the angular resolution is at most $1 / 2 d(v)$.

It remains to show that the drawings produced by the algorithm fit on the $5 n \times 5 n / 2$ grid. Every time we insert a vertex $v_{k}$, we increase the grid size by at most 5 units, which implies that the width of the drawing is at most $5 n$. The final drawing fits inside an isosceles triangle with sides of slope $0,+1,-1$. The width of the base is $5 n$ and so the height is less than $5 n / 2$. 


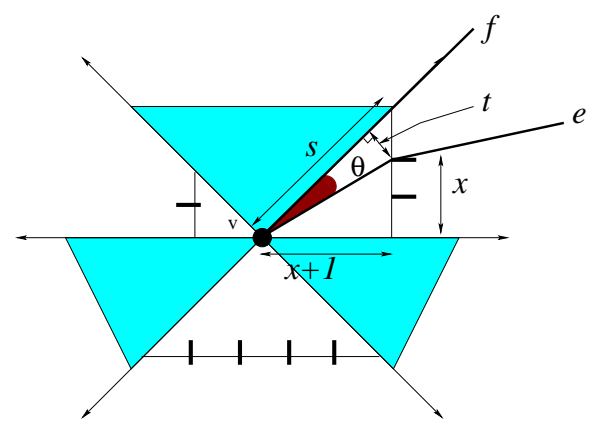

Fig. 4. The minimum angle between two edges adjacent to vertex $v$ is proportional to the degree $d$ of the vertex. Using our algorithm the angle cannot be smaller than $2 / d$.

\section{The PRA Algorithm}

In this section, we introduce a novel approach to representing bends and vertices. Rather than insisting that bends lie on integer grid coordinates, we propose an alternative approach which allows bends to be located on a grid represented by polar coordinates. Using a polar representation allows us to independently control the grid size and edge bend positions. We begin by considering the polar representation in general and then present the PRA algorithm that uses the new approach.

A point $p$ in the polar grid system is represented by a set of integers. For the vertices we only need two integers $\left(p_{x}, p_{y}\right)$. For the bend-points we may need up to five integers. We shall see in the PRA algorithm that these five integers need not be explicitly stored for every bend-point. In general, a bend-point is given by:

$-\left(p_{x}, p_{y}\right)$, the origin of the polar system

$-p_{r}$, the radius of the circle around the origin $\left(p_{x}, p_{y}\right)$

$-p_{d}$ and $p_{n}$, the angle $\left(p_{\theta}\right)$ of the circle where the point is located, i.e., $p_{\theta}=$ $2 \pi p_{n} / p_{d}$. For convenience, we consider $p_{\theta}=0$ to be the vertical direction.

The PRA algorithm places vertices at integer grid coordinates, thus guaranteeing unit vertex resolution. As it is based on the CRA algorithm it also uses only 1 bend per edge. The main difference in the two algorithms is in the placement of the bend-points. In the PRA algorithm, bend-points will be placed on a circle around the vertex (rather than on a straight-line segment). Therefore, the origin, $\left(p_{x}, p_{y}\right)$ for each bend-point need not be explicitly stored - it suffices to store the origin of the vertex that the bend-point is associated with. Similarly, groups of bend-points around a given vertex will have the same radius and hence each of the bend-points need not explicitly store $p_{r}$. Since the points will be evenly spaced in a port region, the values for $p_{\theta}$ need also not be explicitly stored for each bend-point. 


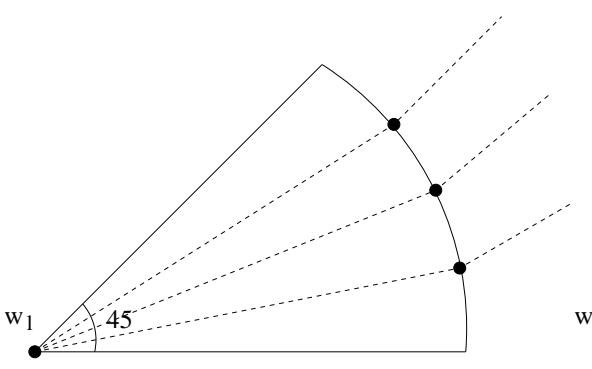

(a)

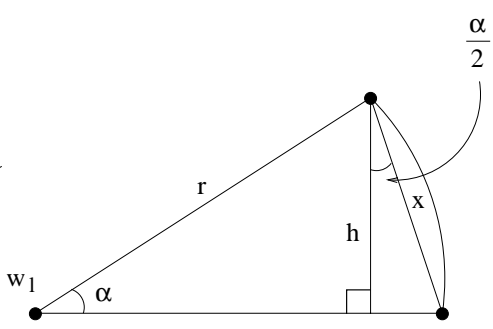

(b)

Fig. 5. Vertex $w_{l}$ is the left-most neighbor of the next vertex $v_{k+1}$ along the exterior face of $G_{k}$. The $d_{r}\left(w_{l}\right)$ ports of $w_{l}$ are evenly spaced on the arc of a circle of radius $2 d_{r}\left(w_{l}\right)$ bounded by the middle free region $M^{f}$ and the right free region $R^{f}$. (a) An example of the layout for the $R^{p}$ region with $d_{r}\left(w_{l}\right)=3$. (b) The distance $x$ between two adjacent ports or a port and an adjacent free region can be computed given the radius of the circle and the angle between the edges connecting the ports to $w_{l}: x=2 r \sin \frac{\alpha}{2}$.

Consider the leftmost neighbor, $w_{l}$, of the next vertex in the canonical order, $v_{k+1}$. The ports are evenly spaced in the $R^{p}$ region for $w_{l}$, Fig. 5(a). The length of the straight-line segment separating two bend-points or a bend-point and an adjacent free region can be computed as follows. Consider the example in Fig. [5(b). We would like to compute the length $x$ in terms of the radius of the circle and the angle between the two line segments connecting consecutive ports to $w_{l}$. From basic trigonometry, the angle between $h$ and $x$ is $\alpha / 2$. We can express $h$ in terms of $r$ and $\alpha: h / r=\sin \alpha$ and we can express $x$ in terms of $h$ and $\alpha$ : $h / x=\cos \alpha / 2$. Combining the two expressions we obtain

$$
x=\frac{h}{\cos \frac{\alpha}{2}}=\frac{r \sin \alpha}{\cos \frac{\alpha}{2}}=\frac{2 r \sin \frac{\alpha}{2} \cos \frac{\alpha}{2}}{\cos \frac{\alpha}{2}}=2 r \sin \frac{\alpha}{2} .
$$

Assume we have inserted $v_{1}, v_{2}, \ldots, v_{k}$ and have a drawing of $G_{k}$ with exterior face $C_{k}$. Consider inserting the next vertex $v_{k+1}$ in the canonical order. Let $w_{l}$ and $w_{r}$ be the leftmost and rightmost neighbors of $v_{k+1}$ on the exterior face $C_{k}$. Define $f_{b}$ and $f_{e}$ to be the bend-point resolution and edge separation respectively. Observe that in the standard Cartesian representation algorithms $f_{b}=1$ and $f_{e}=\sqrt{2} / 2$. Let $d_{r}\left(w_{l}\right)$, respectively $d_{l}\left(w_{r}\right)$, be the number of port edge segments using $R^{p}$ of $w_{l}$, respectively $L^{p}$ of $w_{r}$. When inserting $v_{k+1}$, the degrees for $w_{l}$ and $w_{r}$ affect the amount of shifting necessary to ensure proper resolution. As the cases for $d_{r}\left(w_{l}\right)$ and $d_{l}\left(w_{r}\right)$ are symmetrical, we shall concentrate on $d_{r}\left(w_{l}\right)$. There are two cases to consider:

- case (a) $d_{r}\left(w_{l}\right)=0$ prior to insertion

- case (b) $d_{r}\left(w_{l}\right) \geq 1$ prior to insertion 
In case (a) we insert the first edge in the port region $R^{p}$ between the two free regions $R^{f}$ and $M^{f}$ of $w_{l}$. We place the port in the middle of the arc of a circle connecting $R^{f}$ and $M^{f}$. Since there are no other bends yet in $R^{p}$ we are only concerned with maintaining the edge separation. We need to place the port sufficiently away from the vertex $w_{l}$. Consider the relationship between the radius of the circle and the edge separation, see Fig. 5

The edge separation $f_{e}=x=2 r \sin \frac{\alpha}{2}$. But since there is only one port and it is in the middle of the arc, $\alpha=\pi / 8$. We are interested in the radius necessary to achieve the edge separation $f_{e}$ which is given by

$$
r=\frac{f_{e}}{2 \sin \frac{\alpha}{2}}=\frac{f_{e}}{2 \sin \frac{\pi}{16}}<\frac{4 f_{e}}{\sqrt{2}}=2 \sqrt{2} f_{e} .
$$

Since we maintain that the vertices are at integer coordinates and the radii are also integers, then the minimum radius required in case (a) is

$$
r<\left\lceil 2 \sqrt{2} f_{e}\right\rceil
$$

In case (b) we insert an additional port in the port region $R^{p}$ which already has at least one port. In this case, we must ensure that both the edge separation $f_{e}$ and bend-point resolution $f_{b}$ are preserved. In this case the radius required is given by:

$$
\max \left\{\left\lceil\frac{f_{e}}{2 \sin \frac{\pi}{8\left(d_{r}\left(w_{l}\right)+1\right)}}\right\rceil,\left\lceil\frac{f_{b}}{2 \sin \frac{\pi}{8\left(d_{r}\left(w_{l}\right)+1\right)}}\right\rceil\right\} .
$$

Typically, $f_{b} \geq f_{e}$, so we can assume that the bend-point resolution determines the radius in case (b). Using this together with the fact that $\sin \alpha>0.97 \alpha$ for $\alpha<\pi / 8$, the minimum radius required is

$$
r<\left\lceil\frac{f_{b}}{2 \sin \frac{\pi}{8\left(d_{r}\left(w_{l}\right)+1\right)}}\right\rceil<\left\lceil\sqrt{2} f_{b}\left(d_{r}\left(w_{l}\right)+1\right)\right\rceil
$$

Summing over all vertices in the graph, the sum of the radii used for the right port regions, $R$, yields:

$$
R=\sum_{v_{i} \in V: d_{r}\left(v_{i}\right)=1}\left\lceil 2 \sqrt{2} f_{e}\right\rceil+\sum_{v_{i} \in V: d_{r}\left(v_{i}\right)>1}\left\lceil\sqrt{2} f_{b}\left(d_{r}\left(v_{i}\right)+1\right)\right\rceil .
$$

With $R$ we bounded the number of shifts required because of "right" neighbors. Similarly, we can define $L$, the shifts necessary due to "left" neighbors:

$$
L=\sum_{v_{i} \in V: d_{l}\left(v_{i}\right)=1}\left\lceil 2 \sqrt{2} f_{e}\right\rceil+\sum_{v_{i} \in V: d_{l}\left(v_{i}\right)>1}\left\lceil\sqrt{2} f_{b}\left(d_{l}\left(v_{i}\right)+1\right)\right\rceil .
$$

$L$ and $R$ bound the number of shifts required due to left and right neighbor visibility. Note, however, that if we shift by the minimum amount required by the $f_{e}$ and $f_{b}$ parameters, the location of the next vertex $v_{k+1}$ may not be at integer coordinates. We can guarantee that $v_{k+1}$ is placed on the integer grid 

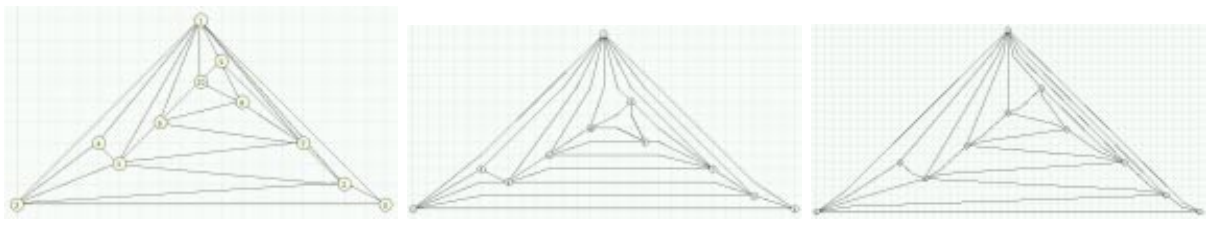

Fig. 6. A graph with 11 vertices drawn using (a) the canonical ordering on the $10 \times 19$ grid; (b) the CRA algorithm on the $14 \times 29$ grid; (c) the PRA algorithm on the $23 \times 45$ grid.
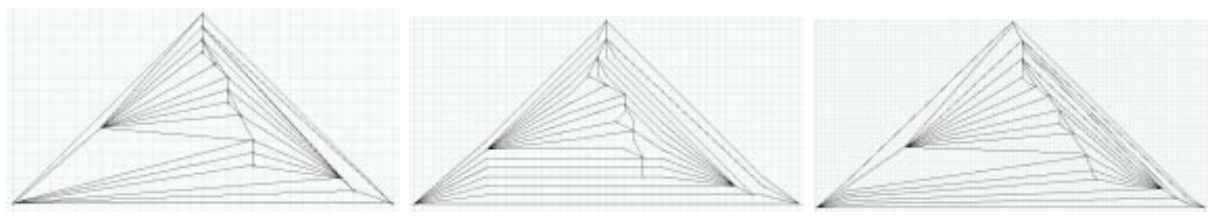

Fig. 7. A graph with 17 vertices drawn using (a) the canonical ordering on the $16 \times 31$ grid; (b) the CRA algorithm on the $21 \times 41$ grid; (c) the PRA algorithm on the $43 \times 85$ grid.

by performing some additional shifts. By shifting at most 3 more units, we are guaranteed to find an integer location for $v_{k+1}$. Then the total shifting required is at most $L+R+3 n$. Since the final drawing fits inside an isoceles right-angle triangle, the total area required for the drawing is $(L+R+3 n) \times\left(\frac{L+R+3 n}{2}\right)$.

In order to compare the PRA algorithm to the CRA algorithm, we evaluate equations 2 and 1 using two sets of parameters, Table 1 In all three cases the algorithms guarantee at most one bend per edge. The PRA algorithms place all the vertices on grid points and each bend-point is determined by at most five integer polar coordinates.

Table 1. Fixing specific values for the vertex resolution $f_{v}$, bend-point resolution $f_{b}$, and edge separation $f_{e}$ allows us to compare the PRA and CRA algorithms.

\begin{tabular}{|c||c|c|c|c|c|}
\hline Algorithm & $f_{v}$ & $f_{b}$ & $f_{e}$ & drawing area & resolution \\
\hline \hline CRA & 1 & 1 & $\sqrt{2} / 2$ & $5 n \times 5 n / 2$ & $1 / 2 d(v)$ \\
\hline PRA1 & 1 & 1 & $\sqrt{2} / 2$ & $9 n \times 9 n / 2$ & $\pi / 4 d(v)$ \\
\hline PRA2 & 1 & $1 / 2$ & $1 / 2$ & $7 n \times 7 n / 2$ & $\pi / 4 d(v)$ \\
\hline
\end{tabular}

\section{Conclusion and Open Problems}

In this paper we present two algorithms for drawing planar graphs with good angular resolution while maintaining small drawing area. Other drawing criteria 
optimized by the algorithms include number of bends, vertex resolution, bendpoint resolution, and edge separation. The first algorithm, CRA, is a traditional algorithm in which vertices and bend-points are represented using Cartesian coordinates. It improves on the best known simultaneous bounds for the six drawing criteria. In the PRA algorithm vertices and bend-points are represented using polar coordinates. It is based on the CRA algorithm but allows for independent control over the grid size and bend positions.

Using a polar coordinate representation yields slightly worse area bounds compared to the CRA algorithm, see Fig [6] and Fig. [7 We believe, however, that the PRA approach is more promising. The angular resolution of the PRA algorithm is better and it provides greater control over the drawing process.

The PRA bounds presented in this paper can be further improved. Using two integers to represent the radius (similar to the way the angles are currently represented) will most likely result in smaller drawing area. Our current estimates indicate that certain (small) values of edge separation and bend-point resolution yield grids of size $4 n \times 2 n$. It is likely that when using only one bend per edge, the best angular resolution will be achieved for vertex regions in which each of the port and free regions have angles $\pi / 3$ rather than a combination of $\pi / 4$ and $\pi / 2$. The biggest challenge, however, to the success of the PRA algorithm deals with the three potential shifts needed to align a new vertex onto an integer grid. If we can reduce this bottleneck, we feel that the PRA algorithm can significantly surpass the bounds of the CRA algorithm.

\section{References}

1. C. C. Cheng, C. A. Duncan, M. T. Goodrich, and S. G. Kobourov. Drawing planar graphs with circular arcs. Discrete and Computational Geometry, 25:405-418, 2001.

2. M. Chrobak and T. Payne. A linear-time algorithm for drawing planar graphs. Inform. Process. Lett., 54:241-246, 1995.

3. H. de Fraysseix, J. Pach, and R. Pollack. How to draw a planar graph on a grid. Combinatorica, 10(1):41-51, 1990.

4. G. Di Battista, P. Eades, R. Tamassia, and I. G. Tollis. Graph Drawing: Algorithms for the Visualization of Graphs. Prentice Hall, Englewood Cliffs, NJ, 1999.

5. A. Garg and R. Tamassia. Planar drawings and angular resolution: Algorithms and bounds. In Proc. 2nd European Symposium on Algorithms, pages 12-23, 1994.

6. M. T. Goodrich and C. G. Wagner. A framework for drawing planar graphs with curves and polylines. In Proc. 6th Symposium on Graph Drawing, pages 153-166, 1998.

7. C. Gutwenger and P. Mutzel. Planar polyline drawings with good angular resolution. In Proc. 6th Symposium on Graph Drawing, pages 167-182, 1998.

8. G. Kant. Drawing planar graphs using the canonical ordering. Algorithmica, 16:4-32, 1996. (special issue on Graph Drawing, edited by G. Di Battista and R. Tamassia).

9. S. Malitz and A. Papakostas. On the angular resolution of planar graphs. SIAM J. Discrete Math., 7:172-183, 1994. 